\title{
Quotients of Infinite Reflection Groups ${ }^{\star}$
}

\author{
Robert L. Griess, Jr.
}

Department of Mathematics, University of Michigan, Ann Arbor, MI 48109, USA

\section{Introduction}

Let $W$ be the Weyl group of a finite dimensional complex simple Lie algebra. The structure of $W$ is quite well-known; see $[2,3]$ for instance. In particular, $W$ is finite and $W / O_{2}(W)$ is isomorphic to a symmetric group or an orthogonal or symplectic group over the field of two elements.

It is natural to consider certain infinite analogues $W(p, q, r)$ of such Weyl groups and inquire about their structure. These are reflection groups defined by diagrams $T(p, q, r)$ of the form

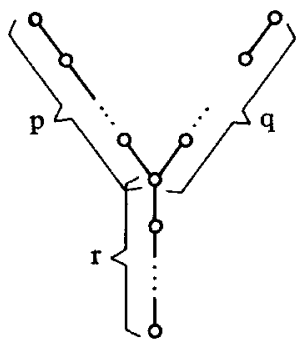

Such groups come up in the theory of Kac-Moody Lie algebras $[10,13]$, the theory of Cremona transformations [7] and in the theory of singularities [11]. Associated to each such diagram is a $W(p, q, r)$-invariant $\mathbb{Z}$-lattice $L=L(p, q, r)$ of rank $p+q+r-2$ with an invariant bilinear form. Fixing a prime number $l$, we let $\bar{W}$ be the image of $W(p, q, r)$ in $G L(L / l L)$. We give the structure of all such $\bar{W}$.

The special case $2=r \leqq p, q=l=2$ was solved by Coble [5]. These are numerous interesting connections with algebraic geometry. See $[4,7]$.

* Research supported by N.S.F. Grant MCS 80-03027, a grant from the University of Michigan and by the John Simon Guggenheim Memorial Foundation 
We should make it clear that our results must not be interpreted as full description of the family of normal subgroups of finite index. There are easy examples of $W$ with normal subgroups not of the form $\operatorname{ker}(W \rightarrow \bar{W})$. Unpublished work of Bernd Fischer gives strong evidence that there is an epimorphism of $W(5,5,4)$ onto $F_{1}$ (the Friendly Giant), a finite simple group of order $2^{46} 3^{20} 5^{9} 7^{6} 11^{2} 13^{3} 17 \cdot 19 \cdot 23 \cdot 29 \cdot 31 \cdot 41 \cdot 47 \cdot 59 \cdot 71$. It would be interesting to have an algebraic geometry interpretation of the kernel of this epimorphism.

It is pleasant that our main results are derived so easily from some existing classification results for finite linear groups. The early result of Coble mentioned above required quite a bit of work. We thank Igor Dolgachev for calling our attention to this problem and providing the above background remarks about algebraic geometry. Reflection groups defined by other diagrams beg to have similar results proven for them. It appears that suitable induction arguments may be tricky to formulate for less familiar classes of diagrams.

\section{Notation and Definitions}

We give notation which will be used throughout the paper. We let $T=T(p, q, r)$ be the diagram

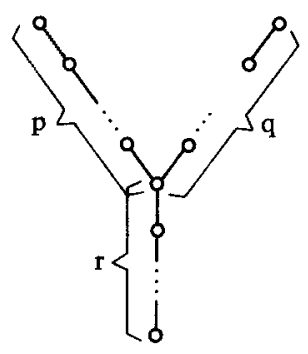

with $n:=p+q+r-2$ nodes, $p, q, r$ all positive. Let $\Phi=\Phi(p, q, r)$ be the vertex set, $L=L(p, q, r)$ the lattice with basis $\Phi$ and symmetric bilinear form $(\alpha, \alpha)=-2$, $(\alpha, \beta)=1$ or 0 as $\alpha, \beta \in \Phi$ are connected or not, $r_{\alpha}: L \rightarrow L$ the reflection $x \mapsto x$ $-(2(x, \alpha) /(\alpha, \alpha)) \alpha=x+(x, \alpha) \alpha$ at $\alpha$ and $W=W_{T}=W(p, q, r)$ the associated reflection group $\left\langle r_{x} \mid \alpha \in \Phi\right\rangle$. Let $A=\Delta_{T}:=\operatorname{det} L$. A deletion of $T$ shall be a connected subdiagram of $T$ with $n-1$ nodes.

Unless $T$ has type $A_{n}, D_{n}$ or $E_{n}, W$ is infinite (see [2]). We aim to determine the quotients $\bar{W}$ of $W$ by its "congruence subgroups".

More precisely, we fix a prime number, $l$. Set $\bar{L}:=L / l L$ and $\bar{W}$ equal to the image of $W$ in $G L(\bar{L})$. Set $\bar{F}=\mathbb{F}_{l}$. We use the bar convention for images in $\bar{L}$ or $\bar{W}$. Since $G L(\bar{L})$ is finite, so is $\bar{W}$. If $l$ is odd, $\bar{W}$ is generated by the reflections $\overline{r_{\alpha}}$, which are -1 on $F \bar{\alpha}$ and 1 on $\bar{\alpha}^{\perp}\left(\bar{L}=F \bar{\alpha} \oplus \bar{\alpha}^{\perp}\right)$. When $l=2$, a different notation is required, that of a transvection (by definition, a nonidentity invertible linear transformation which is trivial on a hyperplane of a vector space and on the associated quotient space).

Let $R(\bar{L})$ be the radical of the form induced on $\bar{L}, d:=\operatorname{dim}_{F}(R(\bar{L})), n^{\prime}:=n-d$, $K:=\operatorname{ker}(\bar{W} \rightarrow G L(\bar{L} / R(\bar{L})), G=\bar{W} / K$. 
For groups $A, B$, we let $A \cdot B$ denote a group with normal subgroups $A$ and quotient $B$. The image of $B$ in $\operatorname{Out}(A)$ shall be understood from the context, although the extension type (i.e. split or nonsplit) of $A \cdot B$ may not be.

Group theoretic notations not explained here may be found in [9].

\section{Preliminary Results}

Lemma 3.1. Every proper submodule of $\bar{L}$ is trivial. All proper submodules generate $R(\bar{L})$. As a $\bar{W}$-module, $\bar{L} / \operatorname{Rad}(\bar{L})$ is irreducible and nontrivial.

Proof. Let $\bar{M}<\bar{L}$ be a nontrivial proper submodule. Then some generator $r=r_{\alpha}$ of $W$ is nontrivial on $\bar{M}$, whence $\bar{M}(1-r) \neq 0$. But $\bar{L}(1-r)=F \bar{\alpha}$. so that $\alpha \in \bar{M}$. Then $\bar{\Phi} \subseteq M$, since two adjacent $\beta, \gamma \in \Phi$ are in a single $W$-orbit by virtue of the equation $\beta\left(r_{\gamma} r_{\beta}\right)=(\beta+\gamma) r_{\beta}=-\beta+(\gamma+\beta)=\gamma$. Since $\bar{\Phi}$ spans $\bar{L}, \bar{L} \leqq \bar{M}$, a contradiction which proves the first assertion.

Now let $\bar{M}<\bar{L}$ be a submodule and take $x \in \bar{M}$. If $(x, \bar{\alpha}) \neq 0$ for some $\alpha \in \Phi, x r_{\alpha}$ $=x+(x, \bar{\alpha}) \bar{\alpha}$ so that $\bar{\alpha} \in \bar{M}$. As above, this is false. So, $\bar{M} \leqq \operatorname{Rad}(\bar{L})$.

Finally, note that $\operatorname{Rad}(\bar{L})<\bar{L}$ since $(\bar{\alpha}, \bar{\beta})=1$ when $\bar{\alpha}, \bar{\beta} \in \Phi$ are adjacent.

Corollary 3.2. $K$ is an elementary abelian l-group, isomorphic to $\underset{1}{\oplus}(\bar{L} / R(\bar{L}))$ as $\bar{W}$-modules, where $d^{\prime}$ is an integer satisfying $0 \leqq d^{\prime} \leqq d$.

Proof. For $k \in K$, we have a linear transformation $T_{k} \in \operatorname{Hom}_{F}(\bar{L} / R(\bar{L}), R(\bar{L}))$ given by $T_{k}: x+R(\bar{L}) \rightarrow x(1-k)$. Using Lemma 3.1 , we get $T_{k_{1}}+T_{k_{2}}=T_{k_{1} k_{2}}$ and $k \mapsto T_{k}$ is a $\bar{W}$-map.

Trivially, the map is injective. So, $\left\{T_{k} \mid k \in K\right\}$ is a $\bar{W}$-submodule of

$$
\operatorname{Hom}_{F}(\bar{L} / R(\bar{L}), R(\bar{L})) \cong \operatorname{Hom}_{F}\left(\bar{L} / R(\bar{L}), F^{d}\right) \cong \bigoplus_{1}^{d} \operatorname{Hom}(\bar{L} / R(\bar{L}), F) \cong \bigoplus_{1}^{d} \bar{L} / R(\bar{L}),
$$

the last isomorphism coming from the self-duality of $\bar{L} / R(\bar{L})$ as a $\bar{W}$-module. Since $\bigoplus_{1}^{d} \bar{L} / R(\bar{L})$ is a semisimple module, the corollary follows.

Dolgachev has called my attention to the following two results.

Lemma 3.3. For any prime $l, \Delta=p q r-p q-q r-r p$.

Proof. Induction.

Lemma 3.4. Let $l$ be arbitrary. Suppose that $\Delta_{T} \equiv 0(\bmod l)$ and $\Delta_{T^{\prime}} \equiv 0(\bmod l)$ for every deletion $T^{\prime}$ of $T$. Then $p \equiv q \equiv r \equiv 0(\bmod l)$. In particular, $p, q, r \geqq l$.

Proof. Use Lemma 3.3.

Corollary 3.5. (i) $d \geqq 1$ if and only if $A \equiv 0(\bmod l)$.

(ii) If $d \geqq 2, d=2 ; d=2$ if and only if $p, q, r \equiv 0(\bmod l)$.

Proof. (i) is obvious.

(ii) Suppose $d>1$; we show that $d=2$ and $p, q, r \equiv 0(\bmod l)$. If $d>1, R\left(\bar{L}_{T^{\prime}}\right) \neq 0$ for every deletion $T^{\prime}$, whence $p, q, r \equiv 0(\bmod l)$, by Lemma 3.4. Again by Lemma 3.4, there is a deletion $T^{\prime \prime}$ of $T^{\prime}=T(p-1, q, r)$ with $\Delta_{T^{\prime \prime}} \neq 0(\bmod l)$. Thus, $0=R\left(\bar{L}_{T^{\prime \prime}}\right)$ $\geqq R(L) \cap L_{T^{\prime \prime}}$, giving $d \leqq 2$ and finally $d=2$. 
Suppose $d=1$. We argue that not all of $p, q, r$ are in $l \mathbb{Z}$. Suppose otherwise. Remove the "middle node" $\beta$ of $T$ to get nonempty diagrams of types $A_{p-1}, A_{q-1}, A_{r-1}$. Let $L_{1}, L_{2}, L_{3}$ be the corresponding lattices. Write $\alpha_{1}^{(1)}, \ldots, \alpha_{p-1}^{(1)}$ for the vertices, in order, of the " $p$-branch" and set $z_{1}=\sum_{k=1}^{p-1} k \alpha_{k}^{(1)}$. Then $\left(z_{1}, \alpha_{1}^{(1)}\right)$ $=-2+2 \cdot 1=0,\left(z_{1}, \alpha_{k}^{(1)}\right)=k-1-2 k+k+1=0$ for $k \in\{2, \ldots, p-2\}$ and $\left(z_{1}, \alpha_{p-1}^{(1)}\right)$ $=p-2-2(p-1)=-p \equiv 0(\bmod l)$. In other words, $\bar{z}_{1}$ lies in radical of $\bar{L}_{1}$. In similar notation, we get $z_{2}=\sum_{k=1}^{q-1} k \alpha_{k}^{(2)}$ and $z_{3}=\sum_{k=1}^{r-1} k \alpha_{k}^{(3)}$ for the other branches. Since $\bar{L}_{0}:=\bar{L}_{1} \oplus \bar{L}_{2} \oplus \bar{L}_{3}$ (orthogonal direct sum) has codimension 1 and $R\left(\bar{L}_{0}\right)$ contains the independent set $\left\{\bar{z}_{1}, \bar{z}_{2}, \bar{z}_{3}\right\}$, we get $d \geqq \operatorname{dim}\left(R\left(\bar{L}_{0}\right) \cap \bar{\beta}^{\perp}\right) \geqq 2$, a contradiction. So, $l$ fails to divide one of $p, q, r$, proving (ii).

Remark 3.6. A bit more work shows that, when $d=2, R(\bar{L})$ is spanned by $z_{1}-z_{2}$ and $z_{2}-z_{3}$.

In order to state the following classification result, we need some definitions. A reflection means a diagonalizable element of some $G L(n, F), \operatorname{char} F \neq 2$, with eigenvalues -1 (once), 1 ( $n-1$ times). For $G \leqq G L(n, F)$, the prefix " $P$ " shall indicate the image of $G$ in $P G L(n, F)$. In the case of a reflection in an orthogonal group, given by the formula $r: x \mapsto x-\frac{2(x, y)}{(y, y)} y$ for some vector $y$, the class of $(y, y)$ in $F^{\times} /\left\{f^{2} \mid f \in F^{\times}\right\}$indicates the conjugacy class of the reflection in the orthogonal group. Thus $R^{\delta} O(V)$ indicates the subgroup of $O(V)$ generated by reflections which correspond to $\delta \in F^{\times}$. Also $R U(V), R L(V)$ denotes the subgroup of $G L(V), G U(V)$ generated by reflections. The superscript " $\varepsilon$ " in $O^{\varepsilon}(n, F)$ indicates the type of quadratic form, as in [1, p. 210]. A wreath product $X Y$ means a semidirect product of $X \times \ldots \times X$ with $Y$, where $Y$ permutes the factors regularly (or "freely"). Finally, $\Sigma L(3,4)$ means $S L(3,4)$ extended by a field automorphism of order 2 .

Proposition 3.7 (Wagner [15], Serežkin and Zalesskii [14]). Let $F$ be a field, $l=\operatorname{char} F \neq 2, V$ a finite dimensional vector space over $F, n=\operatorname{dim} V, G$ an irreducible subgroup of $G L(V)$ generated by reflections. Then $\bar{G}$, the image of $G$ in $P G L(V)$, is one of the following:

(i) $\operatorname{PRL}\left(V^{\prime}\right)$, where $F^{\prime}$ is a subfield of $F, V^{\prime}$ an $F^{\prime}$-subspace of $V$ with $\operatorname{dim}_{F^{\prime}}\left(V^{\prime}\right)=n$.

(ii) $\operatorname{PRU}\left(V^{\prime}\right), V^{\prime}$ as above;

(iii) $P \Sigma L(3,4), n=4, l=3$; here $\bar{G} \leqq P G U(4,3)$;

(iv) $P R^{\delta} O\left(V^{\prime}\right), V^{\prime}$ as above;

(v) $A_{5} \mathbb{Z}_{2}, n=4, l \neq 5 ; \bar{G} \leqq P O^{+}\left(4, F_{l}(\sqrt{5})\right)$;

(vi) $P W_{D_{n}}$ or $P W_{B_{n}}$;

(vii) $2^{4} \cdot \Sigma_{6}$ (nonsplit); $n=4$ and $\sqrt{-1} \in F$;

(viii) $2^{4} \cdot \Sigma_{5}$ (split); $n=4$ and $\sqrt{-1} \in F$;

(ix) $P \Omega(5,3), n=5, l \neq 3, \sqrt[3]{1 \in F \text {; }}$

(x) $P R^{-} O^{-}(6,3), n=6, l \neq 3, \sqrt[3]{1 \in F}$;

(xi) $\mathrm{PO}^{+}(4,3), n=4, l=3$;

(xii) $P O(5,3)$; 
(xiii) $\operatorname{Sp}(6,2), n=7$;

(xiv) $O^{+}(8,2), n=8$

(xv) $\Sigma_{n+1}, l \times n+1$;

(xvi) $\Sigma_{n+2}, \| n+2, n>4$.

Proposition 3.8 (Mclaughlin [12]). Let $G \leqq G L(n, 2)$ be an irreducible subgroup generated by transvections. Then $G \cong O^{\varepsilon}(n, 2)$ ( $n$ even, $\varepsilon=+,-,(n, \varepsilon) \neq(4,2)$ ); $\mathrm{Sp}(n, 2) \cong O(n-1,2)$ (n even); or $\Sigma_{m}$ for $m=n+1$ or $n+2, n$ even.

Also, the conjugacy class of elements of $G$ acting as transvections in some representation is unique, except for $G \cong \Sigma_{6}$ in which there are two such classes.

\section{The Case $l$ odd}

We identify $\bar{W}$, making use of results in Sect. 3. All the "hard work" is contained in Proposition 3.7, with which many possibilities in our situation may be eliminated.

Proposition 4.1. Let $l \neq 2$.

(i) If $T$ has type $A_{n}, D_{n}$ or $E_{n}, \bar{W} \cong W$. Also, $d^{\prime}=1$ for $A_{2}$ and $l=3 ; d^{\prime}=0$ otherwise.

(ii) If Thas type $\tilde{E}_{n-1}, \bar{W} \cong \mathbb{Z}_{l}^{n-1} \cdot W_{T}$, split extension, where $T^{\prime}$ is a deletion of Tof type $E_{n-1}$, respectively. Also $d=d^{\prime}$ unless $T$ has type $\tilde{E}_{6}$ and $l=3$, in which case $d^{\prime}=1, d=2$.

(iii) If $T$ is not "classical" (as in (i) or (ii)), $\bar{W} \cong \mathbb{Z}_{l}^{n^{\prime} d} \cdot G$, split extension, and $G \cong O^{\varepsilon}\left(n^{\prime}, 2\right)$ is an orthogonal group.

Remark 4.2. The invariant $\varepsilon$ may be easily computed from $\Delta_{T^{\prime}}$ and $n^{\prime}$, where $T^{\prime}$ is a subdiagram of $T$ with $n^{\prime}$ nodes and $\left(\Delta_{T^{\prime}}, l\right)=1$; namely, $\varepsilon=+$ if and only if $\Delta_{T^{\prime}}(-1)^{n^{\prime}}$ is a square in $F$ (see $[1$, p. 210]).

Proof. (i) The first statement follows since the kernel of $W \rightarrow \bar{W}$ must be a normal $l$-subgroup of the finite group $W$ and $O_{l}(W)=1$ for such $T$ unless $l=3$ and $T$ has type $A_{2}$. In the latter case, the result may be checked directly.

(ii) Let $T_{0}$ be the normal "translation subgroup" of $W$. By Corollary $3.5, d=1$ or $n=6, l=3, d=2$. We must argue here that $\bar{T}_{0} \neq 1$. However, if $\bar{T}_{0}=1, \bar{W}$ acts like $\bar{W}_{T^{\prime}}$, for an appropriate deletion $T^{\prime}$. But then, $[\bar{L}, \bar{W}]=\left[\bar{L}, \bar{W}_{T^{\prime}}\right]$ has rank at most $n-1$, in contrast with Lemma 3.1. Since $\bar{A}=0$, the only way for $d^{\prime}<d$ to occur is when $d=2$, i.e. type $\tilde{E}_{6}$. There, it is clear that $d^{\prime}=1$.

(iii) Since $T$ is not classical, $n \geqq 8$. Without loss, $p \geqq q \geqq r \geqq 2$ and $p \geqq 4$.

We claim that $G$ is an orthogonal group. We argue by induction on $n$.

If $n=8, p=4$ and $q=r=3$ since $T$ is nonclassical. Here, $\Delta=36-12-12-9=3$. If $l \neq 3$, Proposition 3.7 applies to show that $\bar{W} \cong W_{E_{8}}, \Sigma_{9}, \Sigma_{10}$ or an orthogonal group. Since $\bar{W} \geqq \bar{W}(3,3,3) \cong \mathbb{Z}_{l}^{6} \cdot W_{E_{6}}$, the first three possibilities are out. If $l=3$, $d=1$ and a similar argument with $W(2,3,4) \cong W_{E_{7}}$ shows that $G \cong W_{E_{7}}$ or an orthogonal group. The shape of $T(4,3,3)$ implies that $G$ contains a reflection group of shape $\mathbb{Z}_{2} \times W_{A_{6}}$. So, $W_{E_{7}}$ is out and the claim follows. 
Suppose $n \geqq 9$. If $T$ has a nonclassical deletion $T^{\prime}$, we may use induction, Proposition 3.7 and the fact that $W_{E_{8}}$ and $\Sigma_{n+2}$ do not involve a copy of the orthogonal group $G_{T^{\prime}}$. Now suppose that $T$ has no such deletion.

If $r \geqq 3, q=3$ and $p=4$, against $n \geqq 9$. So $r=2$.

Since $r=2, \Delta=2 p q-p q-2 p-2 q=p q-2(p+q)$. We have $q \geqq 3$, or else $T$ is classical (type $D_{n}$ ). Suppose that $q=3$. Then $p=n-q-r+2 \geqq 6$ and even $p \geqq 7$ or else $T$ has type $\tilde{E}_{8}$. Since every deletion is classical, $p=7$ and $n=10$. Then $\Delta=7 \cdot 3 \cdot 2-7 \cdot 3-7 \cdot 2-3 \cdot 2=1$, so that $\bar{W}$ is irreducible on $\bar{L}$ and $\operatorname{dim} \bar{L}>8$. By Proposition 3.7 either $\bar{W} \cong \Sigma_{11}$ or $\Sigma_{12}$ (impossible, since $W_{E_{8}}$ is not embeddable in $\Sigma_{12}$ ) or it is an orthogonal group, as required. So, $q \geqq 4$. Since every deletion is classical but $T$ is not, $p=5, q=4$ and $r=2$. Then $\Delta=5 \cdot 4 \cdot 2-5 \cdot 4-5 \cdot 2-4 \cdot 2=2$, and $\bar{W}$ operates irreducibly in dimension $n=9$. As above, Proposition 3.7 gives the result.

Our claim that $G$ is an orthogonal group is now verified.

We now claim that $d^{\prime}=d$. Without loss, $\Delta \equiv 0(\bmod l)$. Suppose that $T^{\prime}$ is a deletion with $G_{T} \cong G_{T^{\prime \prime}}$. Since $\left[\bar{L}, W_{T^{\prime}}\right]$ has dimension $n-1, \bar{W}_{T}>\bar{W}_{T^{\prime}}$, and $d^{\prime}>0$. So, we may suppose further that $d=2$. By definition of " $K$ ", $\left[\bar{L}_{T^{\prime}}, K_{T^{\prime}}\right]=R\left(\bar{L}_{T^{\prime}}\right)$. Also, $R\left(\bar{L}_{T^{\prime}}\right) \leqq R\left(\bar{L}_{T}\right)$ for any deletion $T^{\prime}$, since $d=2$.

If $T^{\prime} \neq T^{\prime \prime}$ are deletions and $K_{T^{\prime}} \neq K_{T^{\prime \prime}}$, we are done, as $d^{\prime} \geqq 2$ and $d^{\prime}=2$ follow. So, we may assume that $R\left(\bar{L}_{T^{\prime}}\right)=R\left(L_{T^{\prime \prime}}\right)$. Then, in $\bar{L}^{*}=\bar{L} / \bar{L}_{T^{\prime}} \cap \bar{L}_{T^{\prime \prime}}$, the three nontrivial subspaces are the images of $\bar{L}_{T^{\prime}}, \bar{L}_{T^{\prime \prime}}$, and $R(\bar{L})$. If $X \in\left\{L_{T^{\prime}}, L_{T^{\prime \prime}}\right\}$, a reflection from $X$ in its action on $L^{*}$ preserves the images of $X$ and $R(\bar{L})$, hence of the third space as well. Therefore, $\left[\bar{L}^{*}, \bar{W}\right]=0$, which conflicts with $\bar{L}=[\bar{L}, \bar{W}]$.

To complete the proof that $d^{\prime}=d$, we need to consider the case that every deletion $T^{\prime}$ satisfies $G_{T} \sharp G_{T^{\prime}}$. Then every $T^{\prime}$ is classical, whence $n \leqq 11$. If $n=8$, $p=4$, and $q=r=3=l$. Let $T^{\prime}$ and $T^{\prime \prime}$ be the two deletions of type $E_{7}$ and let $z^{\prime}, z^{\prime \prime}$ be the involutions in $Z\left(W_{T^{\prime}}\right), Z\left(W_{T^{\prime \prime}}\right)$, respectively. Both $z^{\prime}, z^{\prime \prime}$ are -1 on $\bar{L} / R(\bar{L})$, hence map to generators of $Z(G)$. If $d^{\prime}=0, z^{\prime}=z^{\prime \prime} \in Z(W)$, which is certainly false since $\bar{L}=\bar{L}\left(1-z^{\prime}\right) \oplus \bar{L}\left(1+z^{\prime}\right)$ is a submodule decomposition incompatible with Lemma 3.1. So, we may assume that $n \geqq 9$. Then $r=2$ and $(\Delta, l)=1$ (see an earlier paragraph), which is incompatible with $d^{\prime}>0$.

Finally, we show that $1 \rightarrow K \rightarrow \bar{W} \rightarrow G \rightarrow 1$ is always split. Without loss, $\Delta \equiv 0(\bmod l)$. If there is a deletion $T^{\prime}$ with $G_{T^{\prime}} \cong G_{T}$, we use induction. If there is none, every deletion is classical and the analysis of the last paragraph shows that $r \neq 2$. Therefore, $n=8, p=4, q=r=3=l$, and $G \cong W_{E_{7}}$. Let $z$ be an involution of $\bar{W}$ mapping to the element of order 2 in $Z(G)$. Then $C_{\bar{W}}(z) \cong G$ complements $K$. The arguments for (iii) are now complete.

\section{The Case $l=2$}

The analysis here, in spirit, resembles that of Sect. 4. We use Proposition 3.8 here instead of Proposition 3.7. The pleasantness of having a relatively short list of groups in the conclusion of Proposition 3.8 is balanced by the increased complexity involved in determining $G$ and $d^{\prime}$.

In characteristic 2 , we need the notion of a quadratic form as well as that of the bilinear form induced on $\bar{L}$ by the one on $L$. The relevant quadratic form on $L$ is 
$Q(x)=\frac{1}{2}(x, x) \in \mathbb{Z}$. It induces a well defined map $\bar{Q}: \bar{L} \rightarrow F=\mathbb{F}_{2}$ with (,) as its associated bilinear form. See [6] for a discussion of generalities.

Besides the earlier definition of the radical $R(\bar{L})$, we set

$$
R_{\bar{Q}}(\bar{L})=\{\bar{x} \in R(\bar{L}) \mid \bar{Q}(\bar{x})=0\} .
$$

In general, $\operatorname{dim} R(\bar{L}) / R_{\bar{Q}}(\bar{L})=0$ or 1 .

The main results of this section have lengthy statements and are embodied in Lemmas 5.4, 5.5, and 5.6. Notice that $d^{\prime}=d$ is "typical" for $l$ odd, but certainly not so for $l=2$.

Lemma 5.1. Let $L$ be the lattice for $T=T(p, q, 1)$, i.e. type $A_{m}, m=p+q-1$. Let $z=\sum_{k=1}^{m} k \alpha_{k}$, where $\alpha_{1}, \ldots, \alpha_{m}$ is the "natural" order of the roots, i.e. $\alpha_{i}$ and $\alpha_{j}$ are connected if and only if $i-j= \pm 1$. Then, if $m+1 \equiv 0(\bmod l), \bar{z}$ spans $R(\bar{L})$. Also, $(z, z)=-(m+1)$, whatever the congruence of m modulo $l$.

Proof. If $k \in\{2, \ldots, m-1\},\left(\alpha_{k}, z\right)=k-1-2 k+k+1=0$. Also

$$
\left(\alpha_{1}, z\right)=-2+2=0 \text { and }\left(\alpha_{m}, z\right)=m-1-2 m=-1-m \equiv 0(\bmod l) .
$$

So, $\bar{z} \in R(\bar{L}), d \geqq 1$. Since $\Delta(p, q-1,1)=m \neq 0(\bmod 2), d=1$.

We have

$$
\begin{aligned}
(z, z)= & \sum_{k=1}^{m} k^{2}\left(\alpha_{k}, \alpha_{k}\right)+2 \sum_{k=1}^{m-1} k(k+1)\left(\alpha_{k}, \alpha_{k+1}\right)=-2 \frac{m(m+1)(2 m+1)}{6} \\
& +2\left[\frac{(m-1) m(2 m-1)}{6}+\frac{(m-1) m}{2}\right]=-2 m^{2}+m^{2}-m=-m(m+1) .
\end{aligned}
$$

Lemma 5.2. (i) If Thas type $A_{n}, d=0$ or 1 and $d \equiv n(\bmod 2)$, all $n$, and $d^{\prime}=0$ unless $n=3$ in which case $d^{\prime}=1$.

(ii) If $T$ has type $D_{n}, n \geqq 4$, then $d^{\prime}=1, d=1$ or 2 and $d \equiv n(\bmod 2)$; also, $\bar{L} /[\bar{L}, K]$ is isometric to $\bar{L}_{T^{\prime}}$ where $T^{\prime}$ is a deletion of $T$ of type $A_{n-1}$.

Proof. (i) The statement about $d$ follows from Lemma 5.1. Obviously, $d^{\prime}=0$ if $O_{2}\left(W_{A_{n}}\right)=1$, which is the case for $n \neq 1,3$. For $n=1, \bar{W}=1$. For $n=3, d^{\prime} \neq 0$ since $\operatorname{dim}(\bar{L} / R(\bar{L}))=2,|G L(2,2)|=6$, and $\left|W_{A_{3}}\right|=24$.

(ii) We may take $r=q=2$. Then $\Delta=4 p-2 p-2 p-4=-4$ and we use Corollary 3.5 to get the statements about $d$. We get $d^{\prime}>0$ by a simple calculation, e.g. with $\left[2\right.$, p. 256]. So, $d^{\prime}=1$ if $d=1$. Suppose that $d=2$. If $d^{\prime} \neq 1, d^{\prime}=2$, and $U=O_{2}\left(W_{D_{n}}\right)$ has order exceeding $2^{n-1}$ and lies in $K$. So, $n=4$ and $U \cong 2_{+}^{1+4}$ is extraspecial. But $K$ is abelian, by 3.2 , a contradiction.

To prove the second statement, we notice that $[\bar{L}, K] \leqq R_{\bar{Q}}(\bar{L})$; for if $g \in K$ and $y \in \bar{L}, x=y+y^{9}$, then

$$
\bar{Q}(y)=\bar{Q}\left(y^{g}\right)=\bar{Q}(x+y)=\bar{Q}(x)+\bar{Q}(y)+(x, y)=\bar{Q}(x)+\bar{Q}(y)
$$

and $\bar{Q}(x)=0$, as required. Thus, a quadratic form is well-defined on $\bar{L} /[\widetilde{L}, K]$ by taking coset representatives from $\bar{L}_{T^{\prime}}$, a complement to $[\bar{L}, K]\left(\bar{L}_{T^{\prime}}\right.$ is a complement: $K$ is generated by elements which change coordinate signs at two places in 
the usual description of $L$ as in [2],: in that notation, $[\bar{L}, K]=2 L+2 e_{i}$, and our assertion is now clear).

By virtue of Lemma 5.2, we may and do assume $p \geqq q \geqq r \geqq 2$ and $q \geqq 3$ from now through Lemma 5.6.

Lemma 5.3. $G \nsubseteq G L\left(n^{\prime}, 2\right), \Sigma_{m}$.

Proof. Since $G$ lies in an orthogonal group on $\bar{L} / R(\bar{L}), G \nsucceq G L\left(n^{\prime}, 2\right)$ (as $n^{\prime}>2$ ). We show $G \nsubseteq \Sigma_{m}$. If so a subset of the transvections in $\Sigma_{m}$ satisfies $W_{E_{6}}+\Sigma_{7}$, contradiction.

Lemma 5.4. If $(\Delta, 2)=1, G \cong O(\bar{Q})$.

Proof. Since $G \rightarrow O(\bar{Q})$, we apply McLaughlin's Theorem, 3.8, and Lemma 5.3.

Lemma 5.5. Suppose that $\Delta \equiv 0(\bmod 2)$ and $d=1$. Then $n$ is odd and one or three of $p, q, r$ are odd.

If $p, q, r$ are odd,

$$
\bar{W} \cong\left\{\begin{array}{l}
\operatorname{Sp}(n-1,2) \quad \text { for } \quad n \equiv 1(\bmod 4), \\
2^{n-1} \cdot O^{\varepsilon}(n-1,2) \text { for } n \equiv 3(\bmod 4) .
\end{array}\right.
$$

If $\{p, q, r\}=\left\{p_{1}, q_{1}, r_{1}\right\}$ where $p_{1}, q_{1}$ are even and $r_{1}$ is odd,

$$
\bar{W} \cong\left\{\begin{array}{lll}
\operatorname{Sp}(n-1,2) & \text { for } & p_{1}+q_{1} \equiv 2(\bmod 4) \\
2^{n-1} \cdot O^{\varepsilon}(n-1,2) & \text { for } \quad p_{1}+q_{1} \equiv 0(\bmod 4)
\end{array}\right.
$$

In all cases, $\bar{W}$ is a split extension.

Proof. The assertions about $n, p, q, r$ are clear from Lemmas 3.3 and 3.4.

Since $d=1$, there is a deletion $T_{1}$ of $T$ with $\Delta_{1}=\Delta\left(T_{1}\right) \neq 0(\bmod 2)$. We may arrange for $T_{1}=T\left(p_{1}, q_{1}, r_{1}\right)$ to not have type $A_{n-1}$ (easy). Note that $T_{1}$ does not have type $D_{n-1}$ since $\Delta_{1}$ is odd. Without loss, $p_{1} \geqq q_{1} \geqq 3$. If $L_{1}$ and $W_{1}$ are the corresponding lattice and group, $\bar{W}_{1} \cong O^{\varepsilon}(n-1,2)$, by Lemma 5.4. Also, $\bar{L}=[\bar{L}, \bar{W}], \bar{L}_{1}=\left[\bar{L}_{1}, \bar{W}_{1}\right]=\left[\bar{L}, \bar{W}_{1}\right]$, irreducible action of $W_{1}$ on $\bar{L}_{1}$ and Proposition 3.8 imply that $\bar{W} \cong \mathrm{Sp}(n-1,2), 2^{n-1} \cdot \mathrm{Sp}(n-1,2)$ or $2^{n-1} \cdot O^{\varepsilon}(n-1,2)$.

The parities of $n, p, q$, and $r$ imply that there is an extension $S$ of $T$ by one node so that $\Delta(S) \neq 0(\bmod 2)$. Thus, $W(S) \cong O^{\varepsilon}(n+1,2)$ and the embedding $\bar{W} \rightarrow W(S)$ imply that $\bar{W} \cong \mathrm{Sp}(n-1,2)$ or $2^{n-1} \cdot O^{\varepsilon}(n-1,2)$, according to whether $\bar{W}$ stabilizes a nonsingular or singular vector of $\overline{L(S)}$. (Also, $\varepsilon=\varepsilon^{\prime}$ in the latter case). Visibly, the extensions are split.

Case 1. $p, q, r$ odd. Let $\Phi_{1}$ be the nodes of $T$ along the union of the $p$ and $q$-branches and let $z_{1}=\sum_{k=1}^{p+q+1} k \alpha_{k}$ be a sum taken in a natural way along $\Phi_{1}$ (in either direction). Let $\Phi_{2}$ be the $r-2$ outermost roots along the $r$-branch $(r \geqq 3$ here) and let $z_{2}=\sum_{k=1}^{r-2} k \beta_{k}$ be a sum taken along $\Phi_{2}$. Let $L_{1}, L_{2}$ be the corresponding lattices. Then $\bar{z}_{i}$ spans $R\left(\bar{L}_{i}\right), i=1,2$, by Lemma 5.1. Let $\{\delta\}=\Phi-\Phi_{1}-\Phi_{2}$. Then 
$\left(z_{1}, \delta\right) \equiv\left(z_{2}, \delta\right) \equiv 1(\bmod 2)$, whence $R(\bar{L})=F\left(\bar{z}_{1}+\bar{z}_{2}\right)$. Also,

$$
\left(z_{1}+z_{2}, z_{1}+z_{2}\right)=\left(z_{1}, z_{1}\right)+\left(z_{2}, z_{2}\right) \equiv p+q+r-1 \equiv n+1(\bmod 4)
$$

using Lemma 5.1 and the fact that $p+q$ and $r-1$ are even. So, in $\overline{L(S)}, \overline{z_{1}+z_{2}}$ represents a singular, nonsingular vector as $n+1 \equiv 0,2(\bmod 4)$, respectively. Since $\bar{W}$ stabilizes $\overline{z_{1}+z_{2}}$, we get $\bar{W} \cong 2^{n-1} \cdot O^{\varepsilon}(n-1,2)$ or $\mathrm{Sp}(n-1,2)$, accordingly.

Case 2. $r_{1}$ is odd and $p_{1}, q_{1}$ are even. We copy the argument for Case 1, except that we let $\Phi_{2}$ be the $r_{1}-1$ outermost roots of the $r_{1}$-branch. We do not have $\left(L_{1}, L_{2}\right)=0$, though $\left(\bar{z}_{1}, \bar{L}_{2}\right)=0$ since $p_{1} \equiv q_{1} \equiv 0(\bmod 2)$. Thus, $\bar{z}_{1}$ spans $\operatorname{Rad} \bar{L}$. We have $\left(z_{1}, z_{1}\right) \equiv p_{1}+q_{1}(\bmod 4)$, and we finish as before.

Lemma 5.6. Suppose $d=2$. Then $n$ is even and so are $p, q, r$. We have

$$
\left\{\begin{array}{lll}
d^{\prime}=2, & \bar{W} \cong\left(2^{n-2}\right)^{2} \cdot O^{\varepsilon}(n-2,2) & \text { if } \quad p \equiv q \equiv r(\bmod 4) \\
d^{\prime}=1, & \bar{W} \cong 2^{n-2} \cdot \operatorname{Sp}(n-2,2) & \text { otherwise. }
\end{array}\right.
$$

These extensions are all split.

Proof. By Lemma 3.4, all of $p, q, r$ are even. Without loss, $p \geqq q \geqq r$. Let $T_{1}, T_{2}, T_{3}$ be the three deletions of $T$ (at the $p, q$, and $r$ branches, respectively, and let $T_{i j}=T_{i} \cap T_{j}$, $\Delta_{i}=\Delta\left(T_{i}\right), \ldots$ Then $\Delta_{i j}$ is odd. Let $L, W, G, L_{i}, W_{i}, G_{i}$, etc. have the obvious meanings.

Note that there is an enlargement $S$ of $T$ by two nodes (along different branches) so that $\Delta(S)$ is odd. Thus, $\bar{W}$ maps into the stabilizer $H$ in $\overline{W(S)} \cong O^{\varepsilon}(n+2,2)$ of a 2 -dimensional subspace $R(\bar{L})$, which is singular with respect to the bilinear form on $\overline{L(S)}$. Consequently, by Lemma $5.4, G \cong O^{\varepsilon}(n, 2)$ or $\operatorname{Sp}(n, 2)$, as $R_{\bar{Q}}(\bar{L})$ has codimension 0 or 1 in $R(\bar{L})$, respectively.

Suppose $R_{Q}(\bar{L})=R(\bar{L})$. As in Case 2 of Lemma $5.5, \bar{Q}\left(\bar{z}_{i}\right)=0$ and

$$
\left(z_{i}, z_{i}\right) \equiv p_{i}+q_{i} \equiv 0(\bmod 4),
$$

for all $i$. Thus, $p \equiv q \equiv r(\bmod 4)$ and we have $\bar{W}_{i} \cong 2^{n-2} \cdot O^{s_{i}}(n-2,2)$ and $\left\langle\bar{z}_{i}\right\rangle$ $=\left[\bar{L}_{i}, O_{2}\left(\bar{W}_{1}\right)\right]$, for at least two $i$, since $p \geqq q \geqq 4$ (but $r \geqq 2$, only). Let $i$ and $j$ be two such indices. If $\bar{z}_{i}, \bar{z}_{j}$ are linearly independent, we get $d^{\prime}=2$, as required. This is proven by an argument like the one for $d^{\prime}=d$ in Proposition 4.1 (iii).

Suppose $R_{\bar{Q}}(\bar{L}) \neq R(\bar{L})$. We claim that the even integers $p, q, r$ are not pairwise congruent modulo 4. Suppose otherwise. Let $\alpha_{1}^{(i)}, \alpha_{2}^{(i)}, \ldots$ be the roots along the $p, q$, and $r$ branches for $i=1,2,3$, respectively, listed in the inward direction and excluding the node $\beta$ of valency 3 . Set $z_{i}=\sum_{k=1}^{t_{i}-1} k \alpha_{k}^{(i)}, t_{1}=p, t_{2}=\gamma, t_{j}=r$. Then $\left(z_{i}, z_{i}\right)$ $=-t_{i}\left(t_{i}-1\right)$ and $\left(z_{i}, \alpha_{k}^{(j)}\right) \equiv 0(\bmod 2)$ for all $i, j, k$. Since $\left(z_{i}, \beta\right) \equiv 1(\bmod 2)$ for all $i$, $R(\bar{L})$ is spanned by the $z_{i}-z_{j}$. Since

$$
\left(z_{i}-z_{j}, z_{i}-z_{j}\right) \equiv t_{i}+t_{j} \equiv 0(\bmod 4),
$$

we get $\operatorname{dim} R_{\bar{Q}}(\bar{L}) \geqq 2$, against our assumption for this paragraph. Now arrange notation so that $\{p, q, r\}=\left\{p_{1}, q_{1}, r_{1}\right\}, p_{1} \neq q_{1} \equiv r_{1}(\bmod 4)$. If $r>2, p \geqq q \geqq r \geqq 4$, and we argue as follows: By Lemma 5.5 ,

$$
\overline{W\left(p_{1}, q_{1}, r_{1}-1\right)} \cong \operatorname{Sp}(n-2,2), \quad W\left(p_{1}-1, q_{1}, r_{1}\right) \cong 2^{n-2} \cdot O^{\varepsilon}(n-2,2) .
$$


Since $G \cong \operatorname{Sp}(n-2,2)$, this forces $d^{\prime}=1, O_{2}(\bar{W}) \cong 2^{n-2},\left[\bar{L}, O_{2}(W)\right]=\operatorname{Rad}_{Q}(\bar{L})$ and we have the required split extension. Now suppose that $r=2$. Then $n \geqq 8$. If $r=p_{1}$, then

$$
\overline{W\left(p_{1}, q_{1}-1, r_{1}\right)} \cong \overline{W\left(p_{1}, q_{1}, r_{1}-1\right)} \cong \mathrm{Sp}(n-2,2) \cong G
$$

(see the second paragraph of the proof), corcing $d^{\prime} \neq 0$, whence $d^{\prime}=1$ and we have split extension. So, we may assume that $r \neq p_{1}$. Without loss, $r=q_{1}$. Then $r_{1} \equiv 2(\bmod 4)$ implies $r_{1} \geqq 6$, and so $n \geqq 2+4+6-2=10$. The argument for $r>2$ now works. The proof is now complete.

If $\bar{L}$ is nonsingular with respect to the form, the type of the quadratic form is $\varepsilon= \pm$, as the Witt index is maximal of nonmaximal; see [6]. We say that the geometry on $\bar{L}$ has type $\varepsilon= \pm$ if $R(\bar{L})=R_{\bar{Q}}(\bar{L})$ and the type of $(\bar{L} / R(\bar{L}), \bar{Q})$ is $\varepsilon$, and we say that the type is $\varepsilon=0$ if $R(\bar{L}) \neq R_{\bar{Q}}(\bar{L})$. During the previous arguments for $l=2$, the need to know $\varepsilon$ (when $\varepsilon= \pm$ ) was avoided. Here, we give a simple inductive procedure for determining $\varepsilon$ (see Corollary 5.9).

Lemma 5.7. If $L$ is the $A_{4}$-root lattice ( $A_{2}$-root lattice), $\Delta=-5(\Delta=-3)$ and $L / 2 L$ has type $\varepsilon=-$.

Proof. Easily, $\Delta=-5$ (respectively, -3 ). Also, $O^{+}(4,2) \cong \Sigma_{3} \mathbb{Z}_{2}$ (order $2^{3} \cdot 3^{2}$ ) and $O^{-}(4,2) \cong \Sigma_{5}[3,9]$. Since $W\left(A_{4}\right) \cong \Sigma_{5}$ acts faithfully on the lattice modulo $2, \varepsilon=-$. Since $O^{+}(2,2) \cong \mathbb{Z}_{2}$ and $O^{-}(2,2) \cong \Sigma_{3}[3,9]$, similar remarks get $\varepsilon=-$ in this case. An alternate argument is to compute the relevant invariant in the Clifford algebra [6].

Lemma 5.8. Suppose $p \geqq 5$. Let $\alpha_{1}, \alpha_{2}, \ldots$ be the roots on the $p$-branch listed beginning with the outermost one. Let $L_{1}=\sum_{i=1}^{4} \mathbb{Z} \alpha_{i}, \beta=\sum_{k=1}^{5} k \alpha_{k}, L_{2}=\mathbb{Z} \beta+\sum_{\alpha \in \Phi_{1}} \mathbb{Z} \alpha$, where

$$
\Phi_{1}=\Phi-\left\{\alpha_{1}, \alpha_{2}, \alpha_{3}, \alpha_{4}, \alpha_{5}\right\} .
$$

Then $\left(L_{1}, L_{2}\right)=0, \bar{L}=\bar{L}_{1} \oplus \bar{L}_{2}$, and $\bar{L}_{2}$ is isometric to the lattice for $T(p-4, q, r)$ taken modulo 2.

Proof. We have $\left(\alpha_{1}, \beta\right)=-2+2=0,\left(\alpha_{i}, \beta\right)=i-1-2 i+i+1=0$ for $1<i<5$, so that $\left(L_{1}, L_{2}\right)=0$. Also $\beta \in \alpha_{5}+L_{1}+2 L$, whence $\bar{L}=\bar{L}_{1}+\bar{L}_{2}$ and dimension considerations give $\bar{L}=\bar{L}_{1} \oplus \bar{L}_{2}$. Finally, note that if $\alpha \in \Phi_{1},(\alpha, \beta) \equiv\left(\alpha, \alpha_{5}\right)(\bmod 2)$ and $(\beta, \beta)=-5.6$ so that $\bar{Q}(\bar{\beta})=1=\bar{Q}\left(\bar{\alpha}_{5}\right)$; see Lemma 5.1.

Corollary 5.9. If $p \geqq 5, \varepsilon=-\varepsilon^{\prime}$, where $\varepsilon^{\prime}$ is the type of the geometry for $L(p-4, q, r)$ modulo 2.

Proof. The function " $\varepsilon$ " is multiplicative over orthogonal direct sums of nonsingular quadratic spaces over $\mathbb{F}_{2}$.

We now complete our description of the procedure by discussing the "small cases", i.e. $p, q, r$ all less than 5 . They are checked with easy calculations. 
$r=1: n=p+q+1-2=p+q-1, L$ is just the $A_{n}$-lattice, and $\Delta=n+1=p+q$. Since we may as well take $q=1$ here, we may assume that $n=p \leqq 4$. The situation is:

$$
\begin{array}{rr}
n=1 & \varepsilon=0 \\
2 & - \\
3 & - \\
4 & -
\end{array}
$$

$r=2: n=p+q+2-2=p+q, 2 \leqq q \leqq p \leqq 4$. For $q=2$, the lattice is the $D_{n}$-lattice, and the sitaution is the following, according to Lemma 5.2(ii), Corollary 5.9, and the discussion for $r=1$ :

$$
\begin{array}{rr}
n=4 & - \\
5 & - \\
6 & 0
\end{array}
$$

When $q=3$, we get the $E_{6}$-lattice and $\varepsilon=-$ for $p=3$ (use $E_{E_{6}} \cong O^{-}(6,2) \neq O^{+}(6,2$ ) $[3,9]$ ), the $E_{7}$-lattice and $\varepsilon=0$ for $p=4$ (use $W_{E_{7}} \cong \operatorname{Sp}(6,2) \times \mathbb{Z}_{2}[3]$ ). When $q=4=p$, we get the "extended $E_{7}$ "-lattice and $\varepsilon=0$ by noting, as in Lemma 5.2 (ii), that $[\bar{L}, K] \leqq R_{\bar{Q}}(\bar{L})$ and $[\bar{L}, K]$ is complemented by $\bar{L}_{T}$, where $T$ " has type $E_{7}$.

In the remaining case $r=3$ and 4 , we get $\varepsilon$ by observing that when $(\Delta, 2)=1$, the stabilizer in the orthogonal group $O^{\varepsilon}(n, 2)$ of a singular vector looks like $2^{n-2}$ - $O^{\varepsilon}(n-2,2)$.

$r=3: n=p+q+3-2=p+q+1,3 \leqq q \leqq p \leqq 4$. The situation is

$$
\begin{aligned}
(q, p)=(3,3) \quad \varepsilon & =-\quad \text { ("extended } E_{6} " \text {-lattice) } \\
(3,4) \quad \varepsilon=- & \\
(4,4) \quad \varepsilon & =-
\end{aligned}
$$

The case $(3,4)$ may be handled as follows. Here, $\Delta=3$ and the $p$-deletion lends to the group $2^{6} \cdot W_{E_{6}}=2^{6} \cdot O^{-}(6,2)$ and then $\varepsilon=-$, using the observation. The case $(4,4)$ leads to $\varepsilon=-$ via a $q$-deletion, the case for $(3,4)$ and the observation.

$r=4: q=p=4$. A $p$-deletion and the observation gives $\varepsilon=-$.

This concludes the discussion for $l=2$.

\section{Summary of the Structure of $\bar{W}$}

$p \geqq q \geqq r \geqq 1$;

$d=0,1$ or 2 (see 3.5); $\varepsilon$ may be computed using 4.2 for $l \neq 2$ and 5.9 for $l=2$.

$l \neq 2:($ see 4.1$)$

$l=2: r=1, d=0$ or $1, d \equiv n+1(\bmod 2), d^{\prime}=\left\{\begin{array}{ll}0 & n \neq 3 \\ 1 & n=3\end{array}, W \cong \bar{W} \cong W_{A_{n}}\right.$,

$r=2, q=2: d \equiv n(\bmod 2), d^{\prime}=1, W \cong \bar{W} \cong W_{D_{n}}$,

$r=2, q>2$ or $r>2: \Delta \equiv 1(\bmod 2), d=0, \bar{W} \cong O^{\varepsilon}(n, 2)$. 


$$
\Delta \equiv 0(\bmod 2), \quad d=1, \quad p, q, r \text { odd }, \quad \bar{W} \cong \begin{cases}\operatorname{Sp}(n-1,2), & n \equiv 1(\bmod 4), \\ 2^{n-1} \cdot O^{\varepsilon}(n-1,2), & n \equiv 3(\bmod 4)\end{cases}
$$

exactly two of $p, q, r$, say $p_{1}$ and $q_{1}$, are even,

$$
\bar{W} \cong \begin{cases}\operatorname{Sp}(n-1,2), & p_{1}+q_{1} \equiv 2(\bmod 4) \\ 2^{n-1} \cdot O^{\varepsilon}(n-1,2), & p_{1}+q_{1} \equiv 0(\bmod 4)\end{cases}
$$

$d=2, p, q, r$ even,

$$
\left\{\begin{array}{lll}
p \equiv q \equiv r(\bmod 4), & d^{\prime}=2, & \bar{W} \cong\left(2^{n-2}\right)^{2} \cdot O^{\varepsilon}(n-2,2), \\
\text { otherwise } & d^{\prime}=1, & \bar{W} \cong 2^{n-2} \cdot \operatorname{Sp}(n-2,2)
\end{array}\right.
$$

\section{References}

1. Artin, E.: Geometric algebra. New York: Interscience 1957

2. Bourbaki, N.: Groupes et algebres de Lie. Paris: Hermann 1968, Chaps. 4-6

3. Carter, R. Simple groups of Lie type. New York: Wiley 1972

4. Coble, A.: Algebraic geometry and theta functions. A.M.S. Colloq. Publications, X, Providence 1929 (2nd edn. 1961)

5. Coble, A.: Theta modular groups determined by point sets. Am. J. Math. 40, 317-340 (1918)

6. Diendonne, J, : La géometrié des groupes classiques. Berlin, Heidelberg, New York: Springer 1971

7. Dolgachev, I.: Weyl groups and Cremona transformations. Proc. Summer Institute on Singularities, Arcata, 1981 (to appear)

8. Griess, R. : Automorphisms of extraspecial groups and nonvanishing degree 2 cohomology. Pac. J. Math. 48, 403-422 (1973)

9. Gorenstein, D.: Finite groups. New York: Harper and Row 1968

10. Kac, V.G.: Simple irreducible graded Lie algebras of finite growth (Russian). Izv. Akad. Nauk. SSSR 32, 1323-1367 (1968); (English translation) Math. USSR-Izv. 2, 1271-1311 (1968)

11. Looijenga, E.: Rational surfaces with an anticanonical cycle. Ann. Math. 114, 267-322 (1981)

12. McLaughlin, J.E. : Some subgroups of $\mathrm{SL}_{n}\left(\mathbb{F}_{2}\right)$. Ill. J. Math. 13, 108-115 (1969)

13. Moody, R.: A new class of Lie algebras: J. Algebra 10, 211-230 (1968)

14. Serežkin, V.N., Zalesskii, A.E.: Izv. Acad. Sci. USSR Ser. Mat. 44, 1279-1307 (1980)

15. Wagner, A.: Determination of the finite primitive reflection groups over an arbitrary field of characteristic not 2. Parts I-III. Geometriae Dedicata 9, 239-253 (1980); 10, 191-203 (1981); 10, 475-523 (1981)

Received June 14, 1982 